\title{
Efficacy of Conventional Laser Irradiation Versus a New Method for Gingival Depigmentation (Sieve Method): A Clinical Trial
}

\author{
Behzad Houshmand, Noushin Janbakhsh, Fatemeh Khalilian", Mohammad Reza Talebi Ardakani* \\ Department of Periodontics, School of Dentistry, Shahid Beheshti University of Medical Sciences, Tehran, Iran
}

\author{
*Correspondence to \\ Fatemeh Khalilian, D.D.S; \\ Department of Periodontology, \\ Qom University of Medical \\ Sciences, Qom, Iran. \\ Tel: +98-9122078615; Email: \\ fatemehkhalilian1987@gmail.com; \\ Mohammad Reza Talebi Ardakani, \\ D.D.S; Department of Periodontics, \\ Dental School, Shahid Beheshti \\ University of Medical Sciences, \\ Daneshjou Boulevard, Evin, Tehran, \\ Iran. \\ Tel: +98-9122078615. \\ Email:Talebi_m_2002@yahoo.com
}

Published online 28 March 2017

\begin{abstract}
Introduction: Diode laser irradiation has recently shown promising results for treatment of gingival pigmentation. This study sought to compare the efficacy of 2 diode laser irradiation protocols for treatment of gingival pigmentations, namely the conventional method and the sieve method.

Methods: In this split-mouth clinical trial, 15 patients with gingival pigmentation were selected and their pigmentation intensity was determined using Dummett's oral pigmentation index (DOPI) in different dental regions. Diode laser $(980 \mathrm{~nm}$ wavelength and $2 \mathrm{~W}$ power) was irradiated through a stipple pattern (sieve method) and conventionally in the other side of the mouth. Level of pain and satisfaction with the outcome (both patient and periodontist) were measured using a 0-10 visual analog scale (VAS) for both methods. Patients were followed up at 2 weeks, one month and 3 months. Pigmentation levels were compared using repeated measures of analysis of variance (ANOVA). The difference in level of pain and satisfaction between the 2 groups was analyzed by sample $t$ test and general estimate equation model.

Results: No significant differences were found regarding the reduction of pigmentation scores and pain and scores between the 2 groups. The difference in satisfaction with the results at the three time points was significant in both conventional and sieve methods in patients $(P=0.001)$ and periodontists $(P=0.015)$.

Conclusion: Diode laser irradiation in both methods successfully eliminated gingival pigmentations. The sieve method was comparable to conventional technique, offering no additional advantage.
\end{abstract}

Keywords: Gingiva; Pigmentation; Lasers; Semiconductor; Pain; Patient satisfaction.

\section{Introduction}

Healthy oral mucosa is pink in color, but it can vary from light to dark pink depending on the thickness of epithelium, the amount of melanin and the number of erythrocytes present in the connective tissue vessels and their proximity to the surface. Gingival pigmentation is defined as color change of the gingiva from variable shades of pink to dark brown/black due to genetic factors or medication intake. The amount of melanin in melanocytes and adjacent epithelial cells may cause clinical variations in the color of skin and mucosa in different races and ethnic groups. ${ }^{1}$ Such pigmentations are often multifocal or diffuse, and are more commonly seen in darkskinned individuals since infancy. ${ }^{2}$ Gingival pigmentation in these individuals occurs within three hours after birth. ${ }^{3}$ Contrarily, genetic gingival pigmentation does not usually occur in Caucasians such as the Swedish and German populations or the Japanese. Oral pigmentation in these individuals is mainly secondary to smoking, drug intake or some other factors. ${ }^{4}$

Genetics is the most important endogenous factor responsible for increased production of melanin by melanocytes. Some other factors such as the altered activity of endocrine glands and exogenous factors such as UV radiation, smoking and medications may also increase melanin synthesis. The most important drugs causing oral pigmentation include quinolones, antimalarial drugs and minocycline (used for treatment of acne). ${ }^{5}$ Polycyclic amines such as nicotine and benzopyrenes, and free radicals caused by tobacco smoking can also stimulate melanin synthesis by melanocytes. Melanin bonds to these agents to prevent cell injury. The harmless melanin-toxin complex gradually moves towards the superficial epithelial layers and eventually reaches the surface. ${ }^{4}$ In UV-irradiated skin, melanin pigments serve as a trap for toxic products (free radicals) and prevent UV-induced erythema. ${ }^{4}$

According to epidemiologic studies, gingival pigmentation is more common in adults and the most common site

Please cite this article as follows: Houshmand B, Janbakhsh N, Khalilian F, Talebi Ardakani MR. Efficacy of conventional laser irradiation versus a new method for gingival depigmentation (sieve method): a clinical trial. / Lasers Med Sci. 2017;8(2):88-94. doi:10.15171/ jlms.2017.16. 
of involvement in non-smokers is the attached gingiva in the anterior region of the maxilla and mandible followed by the buccal mucosa, lip corners, lip mucosa, dorsal and ventral surface of the tongue and rarely the floor of the mouth. ${ }^{3,4,6}$

Several therapeutic techniques have been proposed and employed for gingival depigmentation. Some of them, such as chemical techniques, are no longer used, ${ }^{7}$ but some others such as gingival abrasion are still practiced. ${ }^{8}$ Cryosurgery $^{9}$ and laser therapy ${ }^{10}$ have also been used for treatment of gingival pigmentation with promising results and have met patients' expectations. Traditional methods for treatment of gingival pigmentation including gingivectomy with free gingival autografting, electro-surgery and radio-surgery have disadvantages such as pain, high cost and risks of surgery, including scar formation, gingival recession, trauma to the bone and periosteum and delayed wound healing.

Laser therapy has been recommended as an alternative modality to the above-mentioned traditional techniques. It has no significant side effects and is extensively applied in medicine and dentistry. ${ }^{11}$

Esthetic dermatology has undergone major advances in the past decades following the introduction of photothermolysis, also known as laser resurfacing. ${ }^{12,13}$ This technique is extensively used to remove fine lines, wrinkles, scars, pigmented areas, and tattoos and has numerous applications for skin rejuvenation and treatment of melasma. Photothermolysis is based on formation of isolated non-contiguous micro-thermal wounds and necrotic zones surrounded by viable tissue in a geometrical pattern, which are not correlated to chromophore distribution. ${ }^{14}$ Diode laser at $810-830 \mathrm{~nm}$ and $980 \mathrm{~nm}$ wavelengths is highly absorbed by the hemoglobin and melanin pigments, which are found in high concentrations in the soft tissue wall of periodontal pockets. Optimal efficacy of diode laser $(980 \mathrm{~nm}, 810 \mathrm{~nm}$ ) for treatment of gingival pigmentation has been documented with no complications. ${ }^{15-17}$ Lower level of pain has often been reported with the use of laser compared to scalpel surgery. ${ }^{18}$ Laser can be irradiated using the conventional method or the recently introduced sieve technique. In the latter technique, laser is irradiated through distinct holes created in a celluloid guide. These holes correspond to the exact location of pigmentations. However, literature is scarce on the efficacy of this technique.

This study sought to compare the efficacy of gingival depigmentation with diode laser $(980 \mathrm{~nm}, 20 \mathrm{~W})$ using the conventional technique versus the sieve method. The null hypothesis was that the level of pain of patients, efficacy of laser therapy and satisfaction of patients and periodontists with the results would be similar following both methods of laser application.

\section{Methods}

This study was conducted on 15 patients (male $=5$, female $=10$, mean age $=33.33 \pm 11.15$ ) with gingival pigmentation, presenting to the Periodontics Department of School of Dentistry, Shahid Beheshti University of Medical Sciences. Patients were selected via convenience sampling. Sample size was calculated to be 15 patients considering $P<0.05$ level of significance, power of $80 \%$, outcome prevalence of $70 \%$ before the intervention and expected reduction in outcome prevalence of $35 \%$ using Minitab software.

Written informed consent was obtained from all patients. A photograph was taken and gingival pigmentation area in the esthetic zone was outlined. The inclusion criteria were: (1) Physiologic gingival pigmentation both at the right and left sides of the mandible and maxilla extending to the distal aspect of the canines, and in form of one continuous ribbon including the entire area between the canines (score 4 in Hedin melanin index). (2) Patient's demand for depigmentation.

The exclusion criteria were: (1) Systemic conditions, medication intake (which is related to medical induced pigmentation) and pregnancy or nursing, (2) Pigmentations linked to malignancy, (3) History of previous depigmentation treatments, (4) Cigarette smoking, and (5) Periodontitis.

Gingival pigmentations were classified into 4 main categories of no pigmentation (index 0), mild light brown tissue (slight pigmentation or index 1), medium brown or mixed brown and pink tissue (moderate pigmentation or index 2) and deep brown/blue-black tissue (severe pigmentation or index 3). Index zero was considered as no pigmentation. The intensity of gingival pigmentation indexes in the right and left sides was determined using the Dummett's oral pigmentation index (DOPI).$^{19}$

After enrolling qualified patients, facial gingival surfaces of patients' incisors were dried using dry compressed air and isolated by cotton rolls. Contralateral quadrants in both the maxilla and mandible were allocated to either of the treatments. Local anesthesia (lidocaine $2 \%$ and epinephrine 1/80.000) was then administered.

In the test side (sieve method), dual diode laser (QuickLase, Canterbury, United Kingdom) was irradiated to pigmented areas in stipple pattern $(980 \mathrm{~nm} 10 \%$ and 810 $\mathrm{nm} 90 \%$, $2 \mathrm{~W}$ with $600 \mathrm{~nm}$ fiber diameter, contact continuous mode). Radiation points were as close as possible to each other, although the fiber was moved enough not to overlap the previous site. In the other side, diode laser was conventionally irradiated at the same session $(980 \mathrm{~nm}$ $90 \%$ and $810 \mathrm{~nm} \mathrm{10 \% ,2} \mathrm{W} \mathrm{power} \mathrm{with} 300 \mathrm{~nm}$ fiber diameter, contact continuous mode). The fiber was moved in brush stroke pattern to cover the whole area. In order to avoid cross over, each quadrant was covered while the other part was being irradiated. The second appointment was scheduled two weeks later to remove the remaining pigmentation if any area was missed. The follow ups were set at 2 weeks, 1 month and 3 months after treatment.

At each follow-up session, a photograph was taken using a digital camera (SLR X16, Canon Corporation, Tokyo, Japan) to compare the outcome of treatment by giving DOPI scores (Figure 1). Gingival pigmentation was assessed by one examiner who was masked to the treatment 

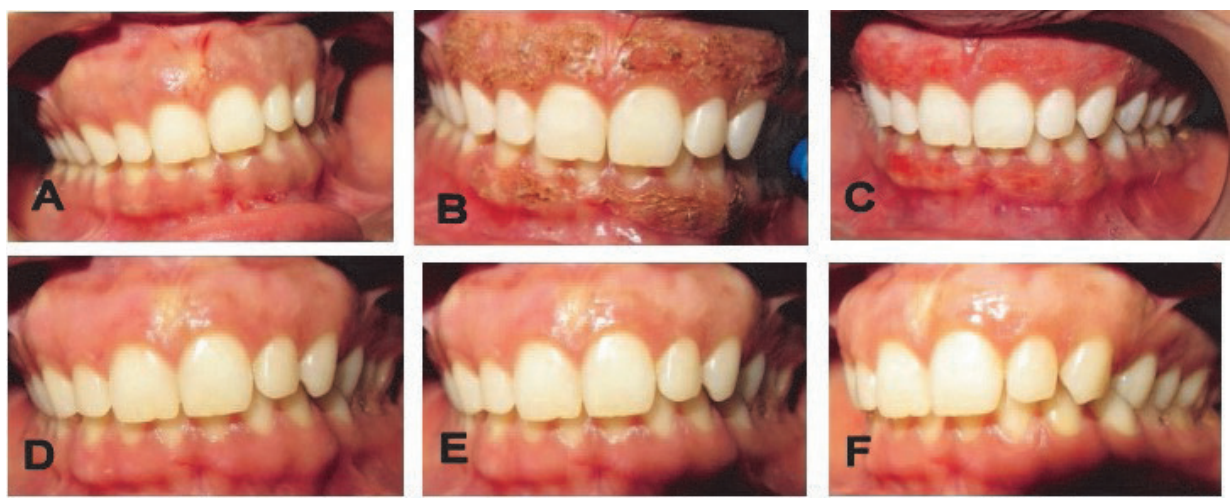

Figure 1. (A) Gingival appearance at baseline. (B) The left side was subjected to the conventional and the right side to the sieve method. (C) Gingival appearance immediately after treatment. (D) Gingival appearance after 2 weeks. (E) Gingival appearance after 1 month. (F) Gingival appearance after 3 months.

procedure. Patients' pain level during the first week after surgery was questioned using a 10 -point visual analog scale (VAS) at the first follow up session. ${ }^{20}$ Level of satisfaction of both patients and practitioners was assessed by a 10-point VAS at each follow up session (2 weeks, 1 month and 3 months).

The data were analyzed using SPSS version 21 (SPSS Inc., IL, USA). The $t$ test was used to ensure that no difference existed between the quadrants at baseline. Paired sample $t$ test was used to compare pain level during the first week after surgery between the 2 methods. Repeated measures analysis of variance (ANOVA) was used to compare DOPI scores of the two treatment methods at all four sessions. General estimating equation (GEE) modeling was used to discover any significant difference in satisfaction of patients and clinicians between the 2 methods, and at different follow-ups. $P<0.05$ was considered significant.

\section{Results}

Fifteen patients were evaluated in this study including 5 females $(33.3 \%)$ and 10 males (66.7\%). The frequency distribution of DOPI classes in central incisor to first molar (teeth \# 1-6) areas in the right and left sides of the mouth is shown in Table 1 (both sides were similar). No significant difference was noticed between the baseline DOPI indices of the 2 groups $(P=1.000)$. Table 2 shows the mean DOPI scores of both groups at baseline and at the follow-ups. Repeated measures ANOVA found no significant difference between the 2 groups at any time point $(P=1.000)$. Significant differences existed between the DOPI scores at baseline and at each of the follow-ups in both groups. However, the difference among the follow up scores was not significant.

Figure 2 shows DOPI changes at the three follow-ups. The values decreased after the treatment and during the follow up sessions; although only the difference between the values at baseline and at each follow up session was significant, and no significant difference existed in the DOPI among the follow-ups.

The mean pain level reported by the patients was $3.47 \pm$ 2.03 in the conventional method and $3.13 \pm 1.59$ in the sieve method. No significant difference was found in the level of pain experienced by patients between the 2 methods (paired sample $t$ test, $P=0.33$ ).

Table 1. The Frequency Distribution of DOPI Classes in Teeth \#13-23 in the Right and Left Sides

\begin{tabular}{|c|c|c|c|c|c|c|c|c|c|c|c|c|}
\hline \multirow{2}{*}{ DOPI Class } & \multicolumn{2}{|c|}{13} & \multicolumn{2}{|c|}{12} & \multicolumn{2}{|c|}{11} & \multicolumn{2}{|c|}{21} & \multicolumn{2}{|c|}{22} & \multicolumn{2}{|c|}{23} \\
\hline & No. & $\%$ & No. & $\%$ & No. & $\%$ & No. & $\%$ & No. & $\%$ & No. & $\%$ \\
\hline No pigmentation (index 0) & 1 & 6.7 & 1 & 6.7 & 1 & 6.7 & 0 & 0 & 0 & 0 & 0 & 0 \\
\hline Mild pigmentation (index 1) & 1 & 6.7 & 1 & 6.7 & 1 & 6.7 & 3 & 20.0 & 3 & 20.0 & 3 & 20.0 \\
\hline Moderate pigmentation (index 2) & 4 & 26.7 & 4 & 26.7 & 4 & 26.7 & 4 & 26.7 & 4 & 26.7 & 4 & 26.7 \\
\hline Severe pigmentation (index 3) & 9 & 60.0 & 9 & 60.0 & 9 & 60.0 & 8 & 53.3 & 8 & 53.3 & 8 & 53.3 \\
\hline
\end{tabular}

Abbreviation: DOPI, Dummett's oral pigmentation index.

Table 2. DOPI Scores of 2 Groups at the Baseline, 2 Weeks, 1 Month and 3 Months Follow ups

\begin{tabular}{lcccc}
\hline & Baseline & 2 Weeks & 1 Month & 3 Months \\
\hline Conventional $($ mean \pm SD) & $2.36 \pm 0.83$ & $0.43 \pm 0.59$ & $0.23 \pm 0.37$ & $0.20 \pm 0.37$ \\
Sieve $($ mean \pm SD) & $2.33 \pm 0.91$ & $0.43 \pm 0.45$ & $0.26 \pm 0.37$ & $0.20 \pm 0.31$ \\
\hline
\end{tabular}

${ }^{a}$ As each treatment was carried out on a separate quadrant (from canine to the central of the same quadrant), the mean DOPI of the 3 tooth in the same quadrant is reported.

Abbreviations: DOPI, Dummett's oral pigmentation index; SD, standard deviation. 


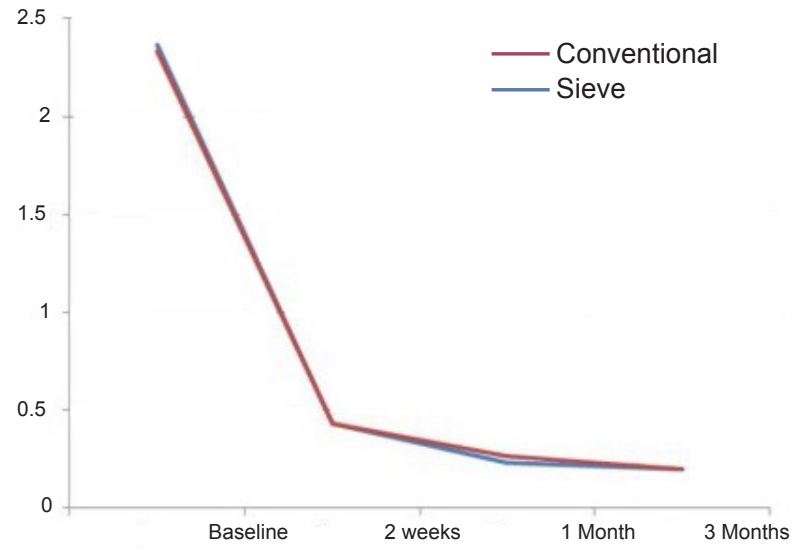

Figure 2. DOPI Level Changes At Baseline, 14, 30 and 90 days follow-ups.

Level of satisfaction of patients and periodontists was assessed using generalized estimating equation. Level of satisfaction in both groups increased after treatment with both methods and there was no significant difference between the 2 methods in this respect $(P=0.93$ for patient satisfaction, $P=0.96$ for periodontist satisfaction). In both groups, significant differences were found between the level of satisfaction at baseline and follow-ups $(P=0.001$ for patients, $P=0.015$ for periodontists). Also, there were significant differences in level of satisfaction at 2 - and 3-month follow ups ( $P=0.005$ for patients, $P=0.007$ for periodontists). Tables 3 and 4 show the satisfaction levels of patients and periodontists, respectively.

\section{Discussion}

In the normal range of gingival color, a wide spectrum of pigmentations exists, depending on the severity of melanogenesis, depth of epithelial rete pegs and gingival vascularization. Melanin; the most common cause of endogenous pigmentations, is synthesized by melanocytes. ${ }^{21}$ Active melanocytes convert tyrosine to melanoprotein (or melanin) via a series of intermediate reactions mediated by tyrosinase. ${ }^{22}$ Melanin is accumulated in melanosomes and transferred to prickle and basal cell layers. Several

Table 3. Satisfaction Levels of Patients With Their Gingival Color at 2 Weeks, 1 Month and 3 Months Follow Ups (reported using a 10 point VAS)

\begin{tabular}{lcccc}
\hline & 2 Weeks & 1 Month & 3 Months & $\boldsymbol{P}$ \\
\hline Conventional & $8.76 \pm 2.25$ & $9.20 \pm 1.56$ & $9.80 \pm 0.77^{*}$ & 0.004 \\
Sieve & $8.53 \pm 2.69$ & $9.20 \pm 1.56$ & $9.80 \pm 0.75^{*}$ & 0.001 \\
\hline
\end{tabular}

*Significant.

Table 4. Satisfaction Levels of Periodontists With Their Gingival Color at 2 Weeks, 1 Month and 3 Months Follow ups (reported using a 10 point $\mathrm{VAS}$ )

\begin{tabular}{lcccc}
\hline & 2 Weeks & 1 Month & 3 Months & $\boldsymbol{P}$ \\
\hline Conventional & $8.87 \pm 2.10$ & $9.20 \pm 1.82$ & $9.60 \pm 0.91^{*}$ & 0.015 \\
Sieve & $8.87 \pm 2.10$ & $9.33 \pm 1.34$ & $9.53 \pm 1.12 *$ & 0.007 \\
\hline
\end{tabular}

*Significant. factors can increase the production of melanin in the mucosa such as trauma, hormones, $\mathrm{x}$-ray radiation and drug intake. $^{23}$

The clinical severity of pigmentation depends on the melanocyte count, number and distribution of melanosomes, degree of melanization of melanosomes, melanin transfer capacity of melanocytes, melanin uptake by keratinocytes and functional activity of melanocytes. ${ }^{24}$

Due to unaesthetic appearance, patients often demand depigmentation treatments. Pigmented layers may be removed by scalpel surgery, bur abrasion, ${ }^{18}$ cryosurgery, ${ }^{25}$ electrosurgery, laser therapy ${ }^{26-28}$ and chemical agents such as $90 \%$ phenol and $95 \%$ alcohol. Pigmented gingiva can also be covered by a free gingival graft ${ }^{29}$ or acellular dermal matrix allografts. ${ }^{30}$

For an effective depigmentation, the majority of melanocytes must be eliminated from the basal layer of gingival epithelium. Non-specific laser irradiation results in ablation of all epithelial layers as well as the rete pegs of the connective tissue, and only remnants of the epithelial rete pegs remain. Er:YAG, $\mathrm{Nd}$ :YAG and $\mathrm{CO}_{2}$ lasers have the necessary requirements for this purpose and are used in dental offices. Thus, they seem to be the modality of choice for it.

In the current study, conventional application of laser in one side and the sieve method in the other side yielded almost similar results with regard to efficacy and satisfaction of patients and periodontists with the outcome of treatment. In other words, application of sieve method had no specific advantage over the conventional technique.

Gingival depigmentation by the use of laser has several advantages over other available techniques. In laser therapy, there is no need to use periodontal dressing after the procedure. Fast healing, short duration of treatment, optimal hemostasis, short healing period, insignificant post-operative pain, no hemorrhage during surgery and optimal antimicrobial activity (similar to sterilization) are among other advantages of gingival depigmentation by laser. The only drawback may be the relatively high cost and the need for special equipment, which may not be available in all clinical dental settings. ${ }^{31}$

Some concerns exist regarding $\mathrm{CO}_{2}$ laser use, due to possible damage to tooth structure. Argon, Er:YAG and Er,Cr:YSGG lasers have been employed with minimal complications and post-operative discomfort; they also enable fast wound healing. Diode laser operating in continuous wave or pulsed mode is a semiconductor solid-state laser recommended for gingival depigmentation. Laser is irradiated with an optical fiber in contact mode, that is, in direct contact with the tissues. Diode laser uses a combination of elements such as gallium, arsenide, aluminum and indium to convert electrical energy into light energy. It has $810 \mathrm{~nm}$ wavelength and thus, it is poorly absorbed by water and highly absorbed by hemoglobin and other pigments. ${ }^{32}$ Diode laser at $810 \mathrm{~nm}$ wavelength enables targeted radiation to soft tissue with excellent coagulation and cutting results. Depigmentation with the use of this laser is achievable due to its penetration into 
hemoglobin and melanin pigments. At low powers, this laser does not affect the dental hard tissue and therefore it is applied as an excellent laser in soft tissue surgery and for cutting and coagulation of gingiva and oral mucosa, as well as for soft tissue curettage or sulcular debridement ${ }^{32}$ with no adverse effect on root surfaces. Diode laser handpiece is small and affordable. Thus, diode laser is used as a safe modality for oral surgery with no damage to dental hard tissue. However, data are scarce on the behavior of melanocytes following surgical trauma due to laser irradiation. Damage to periosteum and alveolar bone is minimal in diode laser irradiation. This laser is capable to eliminate a thin layer of epithelium. Although the healing of laser wounds may be slower than that of scalpel wounds, a sterile inflammatory reaction occurs after laser irradiation. Moreover, surgery with scalpel may cause severe hemorrhage during or after the operation and periodontal dressing must cover the exposed connective tissue for 7 to 10 days. ${ }^{30}$ Diode laser irradiation also has bactericidal effects. Following diode laser irradiation, the adjacent blood vessels are sealed. This process results in hemostasis and creates a dry surgical site. ${ }^{31}$

Lagdive et al, in 2009, compared the results of surgery with scalpel and diode laser and reported that all patients were satisfied with the results of both methods; however, areas irradiated with diode laser had a slower healing process. ${ }^{33}$ They reported that diode laser irradiation was an efficient alternative to scalpel surgery for gingival depigmentation. Mani et al, in 2009, reported that laser irradiation was a safe and efficient treatment for melanin pigmentation of gingiva, with advantages such as easy application, optimal efficacy for treatment of benign superficial pigmented lesions, availability in many dental clinics and minimal trauma. ${ }^{34}$ In 2011, Gupta et al reported the results of gingival depigmentation with diode laser. They reported complete healing at one month and normal pink color of gingiva in the treated area similar to the adjacent normal gingiva. No infection, pain or hemorrhage was reported post-operatively. At 15-months follow up, no recurrence was noted. ${ }^{32}$ In 2012, Kher and Khan evaluated the results of gingival depigmentation with irradiation of diode laser at $980 \mathrm{~nm}$ wavelength in three patients, and reported this modality to be a minimally invasive treatment option for treatment of gingival melanin pigmentation. ${ }^{15}$ In some other studies, optimal efficacy of diode laser irradiation for gingival depigmentation has also been reported. ${ }^{16-18}$ These results are in line with our findings.

The current study had several advantages over the relevant previous ones. Most previous studies on the efficacy of laser for gingival depigmentation have been case reports. However, our study was conducted on 15 patients. Larger sample size increases the reliability of our results compared to those of previous studies. Moreover, we compared the efficacy of 2 methods of laser irradiation, which has not been evaluated before. We also determined the severity of gingival pigmentation in different areas in the right and left sides of the mouth using DOPI, and found no significant difference between the two sides in terms of frequency distribution of DOPI classes. By doing so, we ensured that the 2 sides were matched in terms of severity of melanin pigmentation, and eliminated the confounding effect of different intensities of gingival pigmentation in the two sides on the results. Furthermore, our study had a split-mouth design, which eliminates the effect of possible patient-related confounders on the results. A 10-point VAS was used for assessment of pain intensity and level of satisfaction of patients and periodontists with the results in the 2 methods. No significant difference was noted between the two methods in this regard. In assessment of pain intensity, 1 patient reported a pain score of 8 in the sieve method, while the maximum pain score in the conventional method was 5. Patients were asked to express their level of pain and satisfaction with the results using a $0-10$ point ruler. Slight pain after depigmentation with laser may be related to protein coagulation on the wound surface, serving as a biological barrier. Moreover, laser irradiation seals the sensory nerve ends and inhibits the transfer of pain signals. ${ }^{35}$

Risk of recurrence of gingival pigmentation exists in some cases of depigmentation treatment. According to the migration theory, active melanocytes migrate from the adjacent pigmented tissues to the treated areas and result in recurrence of pigmentation, of which the risk factors are not known. In the current study, patients were followed up for three months and no case of recurrence was noted. This result may be due to the short duration of follow up. Difference in re-pigmentation time may be related to the technique of treatment and race of patients. It may also be related to presence of melanocytes in the areas adjacent to the surgical site. These melanocytes may become activated and synthesize melanin. ${ }^{31}$

In general, the new method introduced in this study presents another laser alternative treatment with satisfactory results. However, it has no superiority over previous laser treatments.

\section{Conclusion}

Within the limitations of this study, no significant difference was noted in the pain score according to VAS between the conventional and sieve methods of laser irradiation for gingival depigmentation. The difference in satisfaction with the results at the three time points was significant in both the conventional and sieve methods in patients and periodontists. No significant differences were noted in terms of the level of patients and periodontists satisfaction with the treatment results at the follow-ups between the 2 techniques. Laser irradiation through sieve method showed comparative results with the conventional method for gingival depigmentation.

\section{Ethical Considerations}

This Study was performed as a clinical trial (IRCTID: IRCT2015111024988N1). This split-mouth triple blind clinical trial was approved by the ethics committee of Shahid Beheshti University of Medical Sciences (IR.SBMU. RIDS.REC.1394.108). 


\section{Conflict of Interests}

The authors declare no conflict of interest, financial or other, exists.

\section{Acknowledgements}

We would like to appreciate Diar Tajhiz Company; the official representator of QuickLase, for their technical support.

\section{References}

1. Wood NK, Goaz PW. Intraoral Brownish, Bluish or Black Conditions. Differential Diagnosis of oral \& Maxillofacial Lesions. St. Louis: Mosby; 1997:182-208.

2. Gondak RO, da Silva-Jorge R, Jorge J, Lopes MA, Vargas PA. Oral pigmented lesions: clinicopathologic features and review of the literature. Med Oral Patol Oral Cir Bucal. 2012;17(6):e919-e924. doi:10.4317/medoral.17679.

3. Newman MG, Takei HH, Carranza FA. Clinical periodontology. 9th ed. Philadelphia: WB. Saunders Co; 2002:30.

4. Hedin CA, Axéll T. Oral melanin pigmentation in 467 Thai and Malaysian people with special emphasis on smoker's melanosis. J Oral Pathol Med. 1991;20(1):8-12. doi:10.1111/j.1600-0714.1991.tb00879.x.

5. Brenner M, Hearing VJ. Modifying skin pigmentation - approaches through intrinsic biochemistry and exogenous agents. Drug Discov Today Dis Mech. 2008;5(2):e189-e99. doi:10.1016/j.ddmec.2008.02.001.

6. Unsal E, Paksoy C, Soykan E, Elhan AH, Sahin M. Oral melanin pigmentation related to smoking in a Turkish population. Community Dent Oral Epidemiol. 2001;29(4):272-277.

7. Hirschfeld I, Hirschfeld L. Oral pigmentation and a method of removing it. Oral Surg Oral Med Oral Pathol. 1951;4(8):1012-1016.

8. Farnoosh AA. Treatment of gingival pigmentation and discoloration for esthetic purposes. Int J Periodontics Restorative Dent. 1990;10(4):312-9.

9. Kumar S, Bhat GS, Bhat KM. Comparative evaluation of gingival depigmentation using tetrafluoroethane cryosurgery and gingival abrasion technique: two years follow up. J Clin Diagn Res. 2013;7(2):389-394. doi:10.7860/JCDR/2013/4454.2779.

10. Azzeh MM. Treatment of gingival hyperpigmentation by erbium-doped:yttrium, aluminum, and garnet laser for esthetic purposes. J Periodontol. 2007;78(1):177-184. doi:10.1902/jop.2007.060167

11. Yousuf A, Hossain M, Nakamura Y, Yamada Y, Kinoshita J, Matsumoto K. Removal of gingival melanin pigmentation with the semiconductor diode laser: a case report. J Clin Laser Med Surg. 2000;18(5):263-266. doi:10.1089/clm.2000.18.263.

12. Altschuler G, Smirnov M, Yaroslavsky I. Lattice of optical islets: a novel treatment modality in photomedicine. J Phys D Appl Phys. 2005;38:2732-2747.

13. Manstein D, Herron GS, Sink RK, Tanner H, Anderson RR. Fractional photothermolysis: a new concept for cutaneous remodeling using microscopic patterns of thermal injury. Lasers Surg Med. 2004;34(5):426-438. doi: 10.1002/lsm.20048.
14. Allen EP, Gladkova ND, Fomina YV, et al. Successful gingival depigmentation with laser-patterned microcoagulation: A case report. Clinic Adv Periodontics. 2011;1(3):210-214. doi:10.1902/cap.2011.110010.

15. Kher U, Khan Z. Gingival depigmentation using the diode laser: three case reports. Int J Laser Dent. 2012;2(3): 7883.

16. Anoop S, Abraham S, Ambili R, Mathew N. Comparative evaluation of gingival depigmentation using scalpel and diode laser with 1 year follow-up. Int J Laser Dent. 2012;2(3):87-91.

17. Singh V, Giliyar SB, Kumar S, Bhat M. Comparative evaluation of gingival depigmentation by diode laser and cryosurgery using tetrafluoroethane: 18-month follow-up. Clinic Adv Periodontics. 2012;2(3):129-134. doi:10.1902/cap.2012.110008.

18. Murthy MB, Kaur J, Das R. Treatment of gingival hyperpigmentation with rotary abrasive, scalpel, and laser techniques: a case series. J Indian Soc Periodontol. 2012;16(4):614-619.

19. Dummett CO, Gupta OP. Estimating the epidemiology of oral pigmentation. J Natl Med Assoc. 1964;56:419-420.

20. Huskisson EC. Measurement of pain. J Rheumatol. 1982;9(5):768-769.

21. Humagain M, Nayak DG, Uppoor AS. Gingival depigmentation: a case report with review of Literature. J Nepal Dent Assoc. 2009;10:53-56.

22. Ciçek Y, Ertaş U. The normal and pathological pigmentation of oral mucous membrane: a review. J Contemp Dent Pract. 2003;4(3):76-86.

23. Tarakji B, Umair A, Prasad D, Alsakran Altamimi M. Diagnosis of oral pigmentations and malignant transformations. Singapore Dent J. 2014;35C:39-46. doi: 10.1016/j.sdj.2014.03.001.

24. Feller L, Masilana A, Khammissa RA, Altini M, Jadwat Y, Lemmer J. Melanin: the biophysiology of oral melanocytes and physiological oral pigmentation. Head Face Med. 2014;10:8.

25. Kathariya R, Pradeep AR. Split mouth de-epithelization techniques for gingival depigmentation: A case series and review of literature. J Indian Soc Periodontol. 2011;15(2):161-168.

26. Atsawasuwan P, Greethong K, Nimmanon V. Treatment of gingival hyperpigmentation for esthetic purposes by Nd:YAG laser: report of 4 cases. J Periodontol. 2000;71(2):315-321.

27. Tal H, Oegiesser D, Tal M. Gingival depigmentation by erbium:YAG laser: clinical observations and patient responses. J Periodontol. 2003;74(11):1660-1667. doi: 10.1902/jop.2003.74.11.1660.

28. Shah C, Dave R, Shah M, Dave D. Evaluation of scalpel versus diode laser for gingival depigmentation: a case report. Int J Adv Health Sci. 2014;1(2):24-27.

29. Tamizi M, Taheri M. Treatment of severe physiologic gingival pigmentation with free gingival autograft. Quintessence Int. 1996;27(8):555-558.

30. Shah SS. Surgical esthetic correction for gingival pigmentation: case series. J Interdiscip Dent. 2012; 2(3):195-200.

31. Kanakamedala AK, Geetha A, Ramakrishnan T, Emadi P. Management of gingival hyperpigmentation by the 
surgical scalpel technique-report of three cases. J Clin Diagn Res. 2010: 14(2):2341-2346.

32. Gupta G. Management of gingival hyperpigmentation by semiconductor diode laser. J Cutan Aesthet Surg. 2011;4(3):208-210. doi:10.4103/0974-2077.91256

33. Lagdive S, Doshi Y, Marawar PP. Management of gingival hyper-pigmentation using surgical blade and diode laser therapy: a comparative study. J Oral Laser Appl. 2009;9:41-47.

34. Mani A, Mani S, Shah S, Thorat V. Management of gingival hyper-pigmentation using surgical blade, diamond bur and diode laser therapy: a case report. J Oral Laser Appl. 2009;9(4):227-232.

35. Hegde R, Padhye A, Sumanth S, Jain AS, Thukral N. Comparison of surgical stripping; erbium-doped:yttrium, aluminum, and garnet laser; and carbon dioxide laser techniques for gingival depigmentation: a clinical and histologic study. J Periodontol. 2013;84(6):738-48. doi: 10.1902/jop.2012.120094. 